\title{
Publisher's Note: Ultimate Precision of Adaptive Noise Estimation [Phys. Rev. Lett. 118, 100502 (2017)]
}

Stefano Pirandola and Cosmo Lupo

(Received 24 August 2017; published 20 September 2017)

DOI: 10.1103/PhysRevLett.119.129902

This Letter was published on 7 March 2017 with an error in the "Note added" section. The Note added should read as "Note added.- Recently, related work [76] appeared on the arXiv." The former endnote [77] and references [78,79] have been removed. This material has been corrected as of 20 September 2017 in the online journal. This material has not been corrected in the printed version of the journal. 